\title{
Investigation of Aerodynamic Performance of High-Speed Train by Detached Eddy Simulation
}

\author{
Hongwei Zheng and Guowei Yang \\ Key Laboratory of High Temperature Gas Dynamics, \\ Institute of Mechanics, Chinese Academy of Sciences, Beijing 100190, China
}

\begin{abstract}
The detached eddy simulation (DES) is employed to study the flows around the high speed train at speed of $500 \mathrm{~km} / \mathrm{h}$. Its object is to evaluate the aerodynamic performance. As we know, for high speed trains at speeds bigger than $250 \mathrm{~km} / \mathrm{h}$, most (about 75-80\%) of the total resistance is caused by aerodynamic drag. Hence, it is important to accurately predict the aerodynamic drag for the designer of new shape of train. To achieve this goal, one of the challenge problems is to capture the massively separations in the wake region which are rarely explored in the literature. To further simplified the problem, the study is only applied to the simplified modelling train which does not include items like bogies, pantographs etc. The results show that DES can capture the separation as well as the small scales of eddies in the wake region.
\end{abstract}

Keywords: High speed train, detached eddy simulation, aerodynamic performance.

\section{Introduction}

The development of high-speed trains is attracting widely attention recently. In the same time, more countries than ever are developing high-speed train operating at speeds of $250 \mathrm{~km} / \mathrm{h}$ and above. There are many aerodynamic-related concerns with the increasing train speed. It includes the aerodynamic drag, the aerodynamically-generated noise, and crosswind stability etc. As we know, for high speed trains at speeds bigger than $250 \mathrm{~km} / \mathrm{h}$, most (about 75-80\%) of the total resistance is caused by aerodynamic drag (Raghunathana et al., 2002). It means, the aerodynamic drag is directly related to the reduction the energy consumption. Hence, the object of this paper is to study the aerodynamic performance especially in the aspect of the aerodynamic drag.

To study the aerodynamic performance, one important way is to use the computational fluid dynamics (CFD) tools. There are several main procedures, Direct Numerical Simulation (DNS), steady (or unsteady) Reynolds Averaged Navier-Stokes (RANS or URANS) simulation, Large-Eddy Simulation (LES) and the hybrid method which combines RANS and LES. For such a high Reynolds number problem with massive separation, it is unaffordable to resolve the small eddies up to the Kolmogrov scale. Hence, the cost is very high if the DNS and LES are applied to this study. For the RANS or URANS, by their very design, it is 
unable to accurately account for the time-dependent and three-dimensional motions governing flows with massive separation. Hence, the DES (Spalart and Allmaras, 1997) is employed to study the turbulent flow over a train at speed of $500 \mathrm{~km} / \mathrm{h}$. It takes advantage of the RANS in the attached regions of the flow and the LES in the unsteady separated regions. It has been proved in a range of challenging test cases which yielding more accurate predictions than can be obtained with RANS. For the high speed trains, it is worth mentioning that complicated flow structures are developed in the wake region behind trains. These wake structures are dominated by large turbulent structures. Most of these structures are resolved using DES and only the influence of the scales smaller than computational cells is modelled. The rest of paper is organized as follows. The numerical method is described in section 2. The flow configuration and the initial mesh are described in section 3. The results are presented in section 4.

\section{Numerical Method: Detached Eddy Simulation}

\subsection{Governing Equations}

To understand the massively separations in the wake region as well as its impact on the time-varying drag force, it is solved by the unsteady Farve-averaged Navier-Stokes equations.

$$
\partial_{t} \int_{\Omega} U d V+\int_{S} \mathrm{~F} \cdot \overrightarrow{\mathrm{n}} d S=0
$$

where $U$ is the state vector, and $F$ is flux vector,

$$
U=\left(\begin{array}{c}
\rho \\
\rho \vec{u} \\
E
\end{array}\right), F=F^{c}-F^{v}, F^{c}=\left(\begin{array}{c}
\rho \vec{u} \\
\rho \vec{u} \otimes \vec{u}+P[\mathrm{I}] \\
(E+P) \vec{u}
\end{array}\right), F^{v}=\left(\begin{array}{c}
0 \\
{[\tau]} \\
([\tau] \cdot \vec{u})+\vec{q}
\end{array}\right) .
$$

Here, $\rho$ is the density, $\vec{u}$ is the velocity, $\tilde{v}$ is the modified viscosity, $\mathrm{E}$ is the total energy, $\mathrm{P}$ is pressure, $[\mathrm{I}]$ is the identity tensor, $[\tau]$ is the stress tensor,

$$
[\tau]=\left(\mu_{L}+\mu_{t u r}\right)\left[\nabla \vec{u}+\nabla^{T} \vec{u}-\frac{2}{3}(\nabla \cdot \vec{u})[\mathrm{I}]\right]
$$

and $\vec{q}$ is heat flux

$$
\vec{q}=\vec{q}^{\text {lam }}+\vec{q}^{\text {turb }}=\left(\kappa_{L}+\kappa_{\text {tur }}\right) \vec{\nabla} T,
$$


with the coefficient

$$
\kappa=\kappa_{L}+\kappa_{t u r}=\frac{\mu_{L} c_{p}}{\operatorname{Pr}_{L}}+\frac{\mu_{t u r} c_{p}}{\operatorname{Pr}_{t u r}} .
$$

The turbulence viscosity in Eq. (3) could be calculated by

$$
\mu_{t u r}=f_{v 1} \rho \tilde{v}
$$

with the dampling function,

$$
f_{v 1}=\frac{\chi^{3}}{\chi^{3}+C_{v 1}^{3}}, \chi=\frac{\tilde{v}}{v_{L}} .
$$

Here, $\tilde{v}$ is calculated by the one-equation Spalart and Allmaras (S-A) turbulence equation (Spalart and Allmaras, 1992),

$$
\begin{aligned}
\frac{\partial}{\partial t} \int_{\Omega} \tilde{v} d \Omega & +\int_{\partial \Omega}\left(\tilde{v} \mathrm{u}-\frac{1}{\sigma_{\tilde{v}}}(v+\tilde{v}) \nabla \tilde{v}-\frac{1}{\sigma_{\tilde{v}}} C_{b 2}(\nabla \tilde{v})^{2}\right) \cdot \vec{n} d S \\
& =\int_{\partial \Omega}\left[C_{b 1}\left(1-f_{t 2}\right) \tilde{S}\right] \tilde{v}-\left(C_{w 1} f_{w}-\frac{C_{b 1}}{\varphi^{2}} f_{t 2}\right)\left(\frac{\tilde{v}}{d}\right)^{2} d S
\end{aligned}
$$

As in the standard DES97 (Spalart et al., 1997), the distance in Eq. (8) are modified as

$$
\tilde{d}=\min \left(C_{D E S} \Delta, d\right)
$$

Here, $\Delta$ is the largest distance between the cell centroid under consideration and the cell centroid of the neighbours. The role of $\Delta$ is to allow the energy cascade down to the grid size; roughly, it makes the pseudo-Kolmogorov length scale, based on the eddy viscosity, proportional to the grid spacing.

\section{$2.2 \quad$ Numerical Discretizations}

Eqs. (1-2) are discretized by using implicit dual-time marching with multistage Runge-Kutta sub-iterations (Weiss and Smith, 1995),

$$
\partial_{t} \int_{\Omega} U d V+\frac{\partial U}{\partial W} \partial_{\tau} \int_{\Omega} W d V+\int_{S} \mathrm{~F} \cdot \mathrm{n} d S=0
$$

with the primitive variables as,

$$
W=\left(\begin{array}{c}
p \\
\vec{u} \\
T
\end{array}\right),
$$


and the Jacobian matrix

$$
\frac{\partial U}{\partial W}=\left[\begin{array}{ccccc}
\rho_{p} & 0 & 0 & 0 & \rho_{T} \\
u_{1} \rho_{p} & \rho & 0 & 0 & \rho_{T} u_{1} \\
u_{2} \rho_{p} & 0 & \rho & 0 & \rho_{T} u_{2} \\
u_{3} \rho_{p} & 0 & 0 & \rho & \rho_{T} u_{3} \\
H \rho_{p}-\left(1-\rho H_{p}\right) & \rho u_{1} & \rho u_{2} & \rho u_{3} & \rho_{T} H+\rho c_{p}
\end{array}\right] .
$$

In each physical time step, the equivalent equations are solved until it reaches a steady state in pseudo time. That is, in each physical time step, we solving the equation until it reach the steady state where the pseudo time derivative term could be neglected.

The set of equation (10) can be discretized at cell $c$ by the multi-stage RungeKutta schemes,

$$
\begin{aligned}
& W_{c}^{(0)}=W_{c}^{m-1} \\
& \ldots \\
& W_{c}^{(i)}=W_{c}^{(0)}-\alpha_{i} \frac{\Delta \tau}{V^{n}}\left[\left(\Gamma_{c}^{-1}\right)^{i-1}+\frac{\Delta \tau}{\Delta t}\left(\frac{\partial U}{\partial W}\right)_{c}\right]^{-1} \operatorname{Res}_{c}^{i-1}, i=1, \ldots, p-1 \\
& \ldots \\
& W_{c}^{m}=W_{c}^{(p)}
\end{aligned}
$$

with the residue defined as

$$
\operatorname{Res}_{c}=\sum_{f} F_{f}\left(U^{-}, U^{+}, \vec{n}\right) \cdot A_{f}+\frac{U_{c}^{i-1} V_{c}^{n}-U_{c}^{n-1} V_{c}^{n-1}}{\Delta t} .
$$

Here, $V_{c}$ is the volume of cell, $F_{f}\left(U^{-}, U^{+}, \vec{n}\right)$ is the ROE numerical flux and $A_{f}$ denotes the area of the face $f$.

\section{Results and Discussions}

In order to study the turbulent flow over a train at the very high speed of $500 \mathrm{~km} / \mathrm{h}$, the simplified high-speed train is used for aerodynamic studies. This simplified model consists of two locomotives (which are named sword and rocket respectively) and one car in the middle. The geometry of the train does not include bogies, rotating wheels, plugs and inter-car gaps (Figure 1). The total length of the train model is $\mathrm{L}=75.5 \mathrm{~m}$. Its height and width are $\mathrm{L}=75.5 \mathrm{~m}$ and $\mathrm{h}=3.42 \mathrm{~m}$ and $\mathrm{w}=2.95 \mathrm{~m}$. The domain is $[-250,250] \times[0,150] \times[-150,150] \mathrm{m}$. The model is shown in Figure 1. 


\begin{tabular}{r|r|r|} 
sword & middle & rocket \\
\hline
\end{tabular}

Fig. 1 Simplified train model

The hybrid mesh (Figure 2) around the train consists of hexahedral viscous boundary layer cell and tetrahedral elements. It is a hybrid unstructured grid and further details are presented below in Table 1. The total number of cells in the computational domain is approximately 15.6 million. This meshing approach made it possible to obtain the necessary spatial resolution.

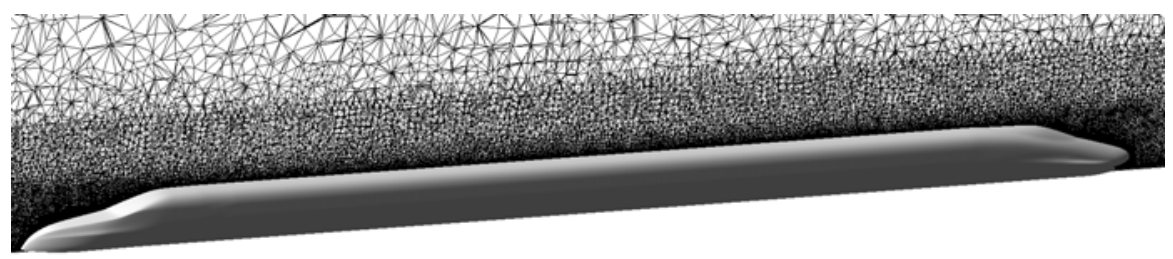

(a) Overview of hybrid mesh around the train

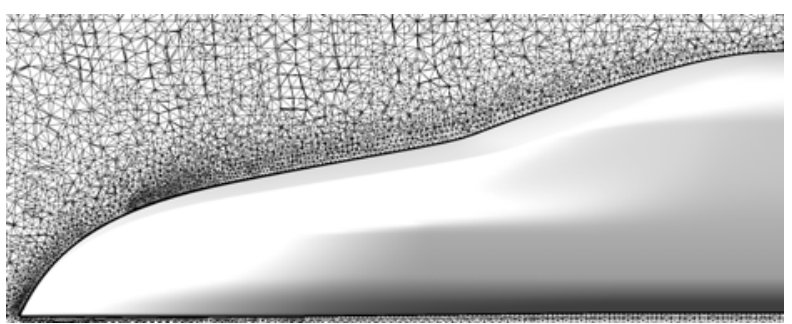

(b) Zoomed view of mesh around the head car

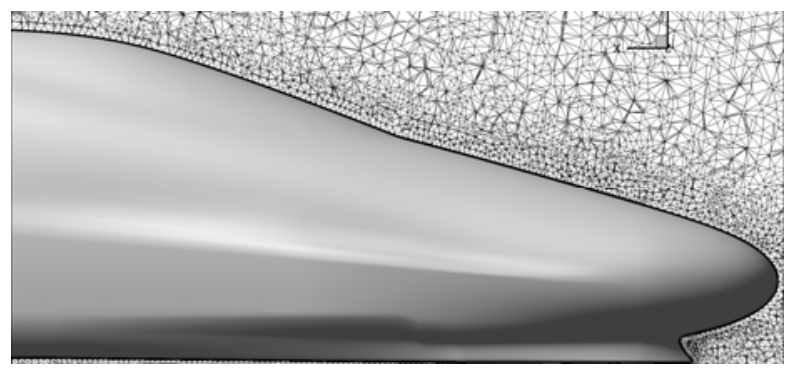

(c) Zoomed view of mesh around the tail car

Fig. 2 Hybrid mesh 
Table 1 Mesh size details

\begin{tabular}{lcccc}
\hline \multirow{2}{*}{$\begin{array}{c}\text { Number of } \\
\text { nodes }\end{array}$} & \multirow{2}{*}{$\begin{array}{c}\text { Number of } \\
\text { elements }\end{array}$} & Brick & Tetrahedral & Pyramid \\
\cline { 3 - 5 } & $15,596,081$ & $4,819,030$ & $10,517,550$ & 259,501 \\
\hline $6,789,704$ & &
\end{tabular}

DES is performed on the previously described model at Reynolds number of $2.29 \times 10^{7}$ based on the free-stream velocity and width of the train. The iso-surface of the instantaneous second invariant of the velocity gradient, $Q=-1 / 2 \partial \mathrm{u}_{\mathrm{i}} / \partial \mathrm{x}_{\mathrm{j}}$ $\partial \mathrm{u}_{\mathrm{j}} / \partial \mathrm{x}_{\mathrm{i}}$, is used to study the temporal evolution of the coherent structures around the train. It is plotted in Figure 3. It shows that the main flow structures in the wake region are two dominant trailing vortex from the skirt and the other two trailing vortex from the nose. Most flows are attached to the boundary. It means the separation mostly occurs in the tail car especially in the region between the nose and the skirt. As compared to the URANS (k- $\omega$ SST) results by StarCCM+, it is easily observed that the DES produces much finer vortex structures. It is primarily due to the excessive dissipation by URANS.

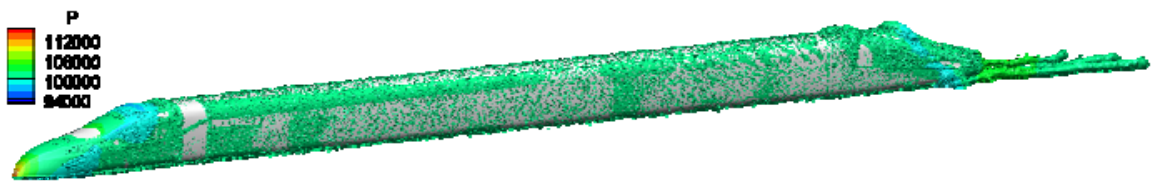

(a) Overview of flows around the train

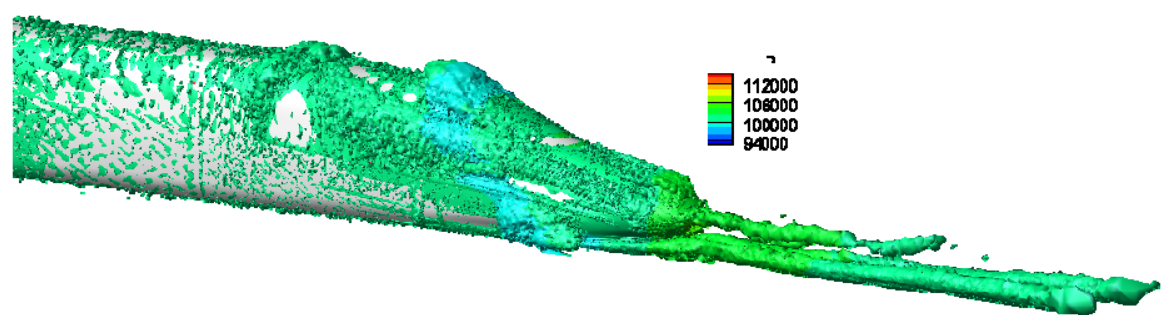

(b) Zoomed view of flows around the tail car

Fig. 3 Instantaneous iso-surface plot of Q-criteria coloured by Pressure

Besides, to be more precise, the quantities such as the drag coefficients of different cars are plotted in Figs. 5-7. Due to a large number of length scales of the vortices being shed from the train, the drag have varying amplitudes of modulations in their time history as presented in figures 5 and 7. It is typical of a massively separated flow field exerting fluctuating forces on the body of disturbance. 


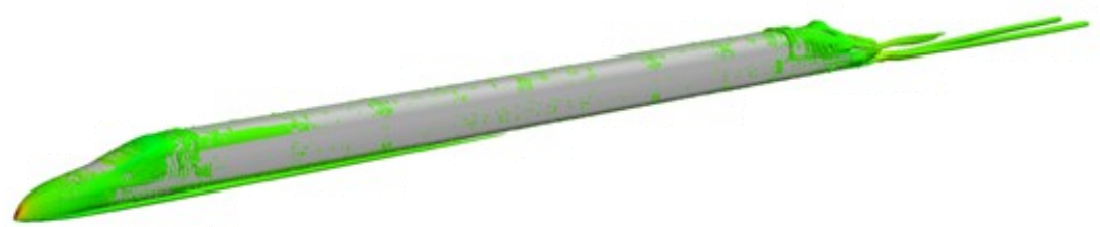

(a) Overview of flows around the train

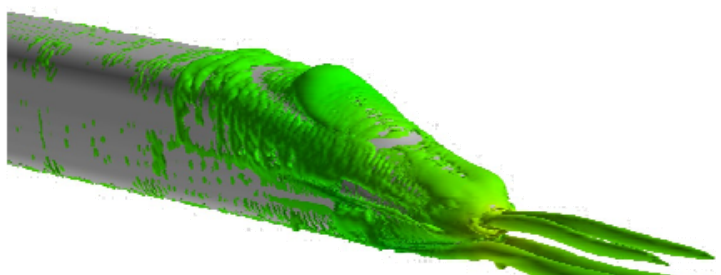

(b) Zoomed view of flows around the tail car

Fig. 4 Instantaneous iso-surface plot of Q-criteria coloured by Pressure (StarCCM+)

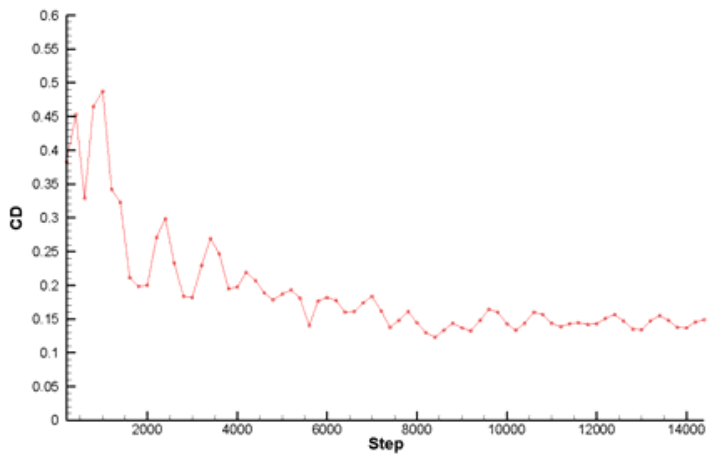

Fig. 5 Time history of $\mathrm{CD}$ of the whole train

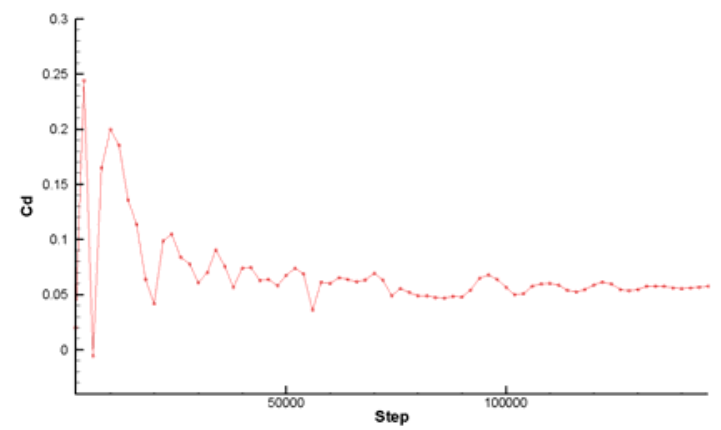

Fig. 6 Time history of CD of the head car (sword) 


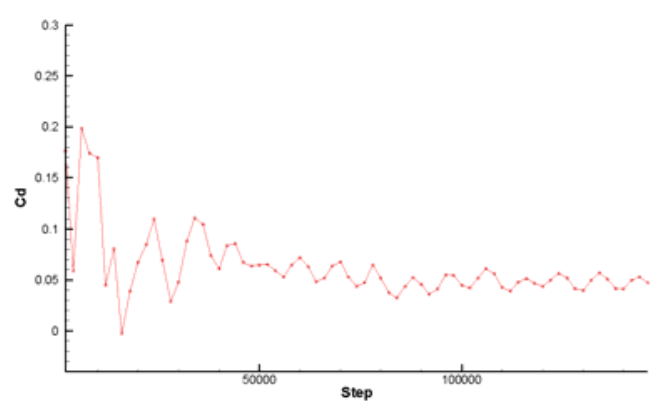

Fig. 7 Time history of $\mathrm{CD}$ of the tail car (rocket)

The averaged drag coefficients for different cars are listed in Table 2. They are compared to the URANS (k- $\omega$ SST) results by StarCCM+ and CFD++ at the speed of $350 \mathrm{~km} / \mathrm{h}$. As for the total drag coefficient, the present result is comparable to those by the URANS. Besides, it is clearly observed that the drag coefficient of the final car is smaller than that of the head car. This is due to the turbulent boundary layer and the small area of wakes developed in the tail car.

Table 2 Total drag coefficients for different cars

\begin{tabular}{ccllll}
\hline & & sword & middle & rocket & Total \\
\hline DES & & 0.057 & 0.040 & 0.048 & 0.146 \\
k- $\omega$ & SST & 0.065 & 0.048 & 0.058 & 0.172 \\
$\begin{array}{l}\text { StarCCM+) } \\
\text { k- } \omega \text { SST (CFD++) }\end{array}$ & & & & 0.1487 \\
\hline
\end{tabular}

\section{Conclusions}

The aerodynamic performance of a simplified high speed train at speed of $500 \mathrm{~km} / \mathrm{h}$ is investigated by detached eddy simulation (DES). It solves the three dimensional unsteady Farve-averaged Navier Stokes equations as well as one algebraic S-A equation by our in-house dual-time step finite volume solver. It shows that the main flow structures in the wake region are two dominant trailing vortex from the skirt and the other two trailing vortex from the nose. As compared to the URANS (k- $\omega$ SST) results by StarCCM+ and CFD++, the results by the DES produce much finer vortex structures. Besides, the drag coefficient of the final car is smaller than that of the head car. 


\section{References}

Raghunathana, R.S., Kim, H.-D., Setoguchic, T.: Aerodynamics of high-speed railway train. Progress in Aerospace Sciences 38(6-7), 469-514 (2002)

Spalart, P.R., Jou, W.-H., Strelets, M., Allmaras, S.R.: Comments on the feasibility of LES for wings, and on a hybrid RANS/LES. In: Proceedings of the 1st AFOSR International Conference on DNS and LES, pp. 137-147. Greyden Press (1997)

Spalart, P.R., Allmaras, S.R.: A One-Equation Turbulence Model for Aerodynamic Flows. AIAA Paper 92-0439 (1992)

Weiss, M., Smith, W.A.: Preconditioning applied to variable and constant density flow. AIAA J. 33, 2050-2057 (1995) 